\title{
EVALUACIÓN INSTRUMENTAL COLORIMÉTRICA DE RESINAS COMPUESTAS QUE IMITAN DENTINA EN COMPARACIÓN A ESCALA VITA CLÁSICA
}

\section{INSTRUMENTAL COLORIMETRIC EVALUATION OF COMPOSITE RESINS THAT MIMIC DENTIN BY A VITA CLASSIC SCALE COMPARISON}

\author{
Sarmiento Jaime ${ }^{1}$, Morales Jessie $^{2}$, Hidalgo Leslie ${ }^{3}$, Leiva Iván ${ }^{3}$
}

\section{RESUMEN}

Objetivos: Determinar cuantitativamente en 7 marcas comerciales diferentes de resinas compuestas que simulan dentina (Filtek Z350XT® 3M ESPE TM, Miris $2 \circledR$ Coltène $^{\mathrm{TM}}$, Empress Direct ${ }^{\circledR}$ Ivoclar Vivadent ${ }^{\mathrm{TM}}$, Opallis $\AA$ $\mathrm{FGM}^{\mathrm{TM}}$, TPH Spectra ${ }^{\circledR}$ DentsplySirona TM Tetric N Ceram ${ }^{\circledR}$ Ivoclar Vivadent ${ }^{\mathrm{TM}}$, Brilliant Everglow ${ }^{\circledR}$ Coltène ${ }^{\mathrm{TM}}$ ) codificado como Vita Clásica Vita Zahnfabrik ${ }^{\mathrm{TM}}$ ) A2 y A3 o un tinte equivalente, la diferencia de color $(\Delta E)$ con la escala Vita Classic para esa codificación en un fondo blanco o negro

Materiales y métodos: Estudio descriptivo in vitro. Se utilizaron 7 marcas de resinas compuestas ( $N$ 35) en tonos $\mathrm{A} 3$ y $\mathrm{A} 2$, se realizaron discos de $10 \mathrm{~mm}$ de diámetro y $2 \mathrm{~mm}$ de grosor, medidos con espectrofotometría en 2 fondos con instrumento Vita Easyshade $\mathrm{V} \circledast$ Vita Zahnfabrik ${ }^{\mathrm{TM}}$. Se analizaron los valores obtenidos en CIELAB obteniendo $\triangle \mathrm{E}$. Se realizó una prueba ANOVA 2 factores $(\mathrm{a} 0,05)$.

Resultados: El $\Delta \mathrm{E}$ más cercano a la escala Vita Clásica $\AA^{\circledR}$ fue dado por TPH Spectra ${ }^{\circledR}$ DentsplySirona ${ }^{\mathrm{TM}}$ A2 $(1.02 \pm .69)$, el $\triangle \mathrm{E}$ mayor fue de Filtek Z350XT® 3M $\operatorname{ESPE}^{\mathrm{TM}}(69.31 \pm .50)$.

Conclusión: Existen claras diferencias entre las resinas de las marcas comerciales estudiadas y la escala Vita Clásica. En fondo negro un $71,4 \%$ de todas las muestras y en fondo blanco un $92,8 \%$ están sobre un $\Delta \mathrm{E} 5$, significando que las diferencias entre sus tintes son consideradas inaceptables clínicamente. Ninguna de las marcas de resinas compuestas cumple con las características colorimétricas al compararlas con la escala Vita Clásica.
1. Cátedra de Operatoria Dental, Escuela de Odontología, Universidad de Valparaíso, Chile. Servicio Odontológico de la Armada de Chile.

2. Centro de Salud Familiar Las Cañas, Valparaíso, Chile.

3. Universidad de Valparaíso, Chile.

\section{Correspondencia:}

Jaime Sarmiento

Escuela de Odontología. Subida Leopoldo Carvallo \#211, Valparaíso, Chile.

Correo electrónico:

jaime.sarmiento@uv.cl

\section{PALABRAS CLAVES:}

Resina compuesta; Color; Dentina; Espectrofotometría; Estética dental.

\section{KEYWORDS:}

Composite; Color; Dentin;

Spectrophotometry; Dental aesthetics. 


\section{ABSTRACT}

Objective: To quantitatively determine in 7 different commercial brands of composite resins that mimic dentin (Filtek Z350XT® 3M ESPE ${ }^{\mathrm{TM}}$, Miris $2 \circledast$ Coltène ${ }^{\mathrm{TM}}$, Empress Direct $\circledast$ Ivoclar Vivadent $^{\mathrm{TM}}$, Opallis ${ }^{\circledR} \mathrm{FGM}^{\mathrm{TM}}$, TPH Spectra ${ }^{\circledR}$ DentsplySirona ${ }^{\mathrm{TM}}$ Tetric $\mathrm{N}$ Ceram ${ }^{\circledR}$ Ivoclar Vivadent $^{\mathrm{TM}}$, Brilliant Everglow ${ }^{\circledR}$ Coltène ${ }^{\mathrm{TM}}$ ) coded as Vita Clásica Vita Zahnfabrik ${ }^{\text {TM's }}$ A2 and $\mathrm{A} 3$ or an equivalent dye, the difference in color $(\Delta \mathrm{E})$ with the Vita Classic scale for that codification on a black or white background.

Materials and methods: In vitro descriptive study. 7 composite resin brands (N 35) in A3 and $\mathrm{A} 2$ tones were used, $10 \mathrm{~mm}$ diameter and 2 $\mathrm{mm}$ thick discs were made, measured with spectrophotometry on two backgrounds with Vita Easyshade V® Vita Zahnfabrik TM instrument. The values obtained in CIELAB were analyzed obtaining $\triangle \mathrm{E}$. An ANOVA 2 factor test (a0.05) was performed.

Results: The $\Delta \mathrm{E}$ closest to the Vita Classic $\AA$ scale was given by TPH Spectra ${ }^{\circledR}$ DentsplySirona TM A2 (1.02 \pm .69$)$, the largest $\triangle \mathrm{E}$ was Filtek Z350XT® $3 \mathrm{M}$ ESPE TM (69.31 \pm $.50)$.

Conclusion: There are clear differences between composite resins of the studied brands and the Vita Classical scale. On a black background $71.4 \%$ of all samples and on a white background $92.8 \%$ are on a $\Delta \mathrm{E} 5$, meaning that the differences between their dyes are considered clinically unacceptable. None of the composite resin brands meet the colorimetric characteristics when compared to the Vita Classic scale.

\section{INTRODUCCIÓN}

Hoy en día los pacientes le solicitan al odontólogo que aparte de recuperar la función, otorguen una estética en los diversos tratamientos que se pueden realizar, esto a través de los distintos materiales y técnicas.

Dentro de los objetivos principales de la estética en odontología es que, al restaurar la estructura pérdida de un diente, se recupere su función, forma $\mathrm{y}$ apariencia original. Para esto se necesitan conocimientos de anatomía dentaria, textura superficial, translucidez y color de la restauración.

Uno de los problemas a los que se ve enfrentado el profesional es el de seleccionar el color dentario. El color es un proceso físico y neurofisiológico de la visión, asociado con las diferentes longitudes de onda en la zona visible del espectro electromagnético. ${ }^{1}$ La percepción del color es una respuesta fisiológica a un estímulo físico. ${ }^{2}$ Se debe hacer diferencia entre esmalte y dentina.

El mercado de materiales dentales ofrece una gran diversidad de sistemas de resina compuesta directa, pero no existe una estandarización en la codificación cromática entre las distintas compañías fabricantes, esto genera muchas dificultades para elegir que material usar. La compañía $V$ ita $^{\mathrm{TM}}$ y su nomenclatura ha sido utilizada como referente por diferentes fabricantes como base para codificar sus distintos tonos de resina compuesta, es por esto que surge la necesidad de determinar si existen diferencias colorimétricas entre las jeringas de resina compuesta que asemejan dentina de distintas marcas codificadas con el sistema Vita. El tono, tinte o matiz es el estado puro del color, sin el blanco o negro agregados. En la guía Vita clásica, podemos reconocer 2 tintes: marrón con sus variantes A y D; y amarillo con su variante $B$. El C corresponde a un $\mathrm{B}$ con modificaciones adicionales de valor. $\mathrm{C}$ es su porción acromática. ${ }^{3}$

Es por esto que el objetivo es determinar manera cuantitativa en las líneas comerciales, Filtek Z350XT ${ }^{\circledR} 3 \mathrm{M}$ ESPETM $^{\mathrm{TM}}$, Miris 2 ${ }^{\circledR}$ Coltène $^{\mathrm{TM}}$, Empress Direct ${ }^{\circledR}$ Ivoclar Vivadent ${ }^{\mathrm{TM}}$, Opallis ${ }^{\circledR}$ FGM $^{\mathrm{TM}}$, TPH Spectra ${ }^{\circledR}$ DentsplySirona ${ }^{\mathrm{TM}}$ Tetric $\mathrm{N} \quad$ Ceram $^{\circledR} \quad$ Ivoclar Vivadent ${ }^{\mathrm{TM}}$, Brilliant Everglow ${ }^{\circledR}$ Coltène ${ }^{\mathrm{TM}}$, que simulan dentina, codificada en A2 y A3 de Vita Clásica Vita Zahnfabrik $^{\mathrm{TM}}$ o equivalente, la diferencia del color $(\Delta \mathrm{E})$ con la escala Vita Clásica para esa codificación en diferentes fondos blanco y negro. La diferencia de color o delta $\mathrm{E}(\Delta \mathrm{E})$ define la diferencia (visual y/o medible) entre el color obtenido con respecto al color de referencia y se indica como la distancia entre las coordenadas del color obtenido y las coordenadas del color de referencia. 4 


\section{MATERIALES Y MÉTODOS}

El estudio correspondió a un estudio de tipo descriptivo in vitro sin muestras biológicas, basado en la elaboración y observación de muestras de resina compuesta, donde se utilizaron siete marcas diferentes en dos tonos, A3 y A2.

El universo abarca a los biomateriales de resinas compuestas de simulación de dentina de los sistemas de compañías fabricantes para técnica de restauración directa.

Se utilizó la proporción de coincidencia de la muestra completa, es decir, considerando a todas las resinas compuestas.

Se determinó utilizar un tamaño muestral de $n=5$ por marca comercial bajo la fórmula de tamaño muestral para determinar parámetros (ver anexo I), con una sola medición por cuerpo de prueba considerando que, se efectúo un testeo previo de varias mediciones por cuerpo de prueba, sin diferencias significativas. Esto dio un total de 35 cuerpos de prueba para cada tono.

Los cuerpos de prueba fueron confeccionados en forma de disco, sobre una matriz plástica construida en impresora 3D, con $10 \mathrm{~mm}$ de diámetro y $2 \mathrm{~mm}$ de grosor. La aplicación de las resinas compuestas sobre el conformador fue homogénea, este procedimiento se realizó utilizando una espátula de resina compuesta y en un solo incremento, con el fin de limitar la posibilidad de incorporación de burbujas de aire que alteren las lecturas proporcionadas por el espectrofotómetro. La polimerización fue realizada en función de una unidad de fotocurado Led modelo Valo ${ }^{\circledR}$ de Ultradent ${ }^{\mathrm{TM}}$, a intensidad extra de $3.200 \mathrm{~mW} / \mathrm{cm}^{2}$, durante que el tiempo que indicó el fabricante. Las muestras se midieron con calibrador de metales Germany $\mathrm{CE}$ Inox para corroborar su espesor, incluyéndose en el estudio aquellas que tuvieran una diferencia no mayor a $+/-0,1 \mathrm{~mm}$ respecto del espesor y diámetro definido. La confección de las muestras fue realizada por un solo operador, el cual previamente fue capacitado. Los cuerpos de prueba fueron almacenados durante una semana a temperatura ambiente, en un sitio oscuro dentro de bolsas plásticas transparentes.
El muestreo fue seleccionado por conveniencia, incluyendo este estudio sólo aquellos cuerpos de prueba que estuviesen indemnes, y se excluyeron los que presentaron fracturas o algún daño. El universo de este estudio corresponde a los biomateriales de resinas compuestas de simulación de dentina de los sistemas de compañías fabricantes para técnica de restauración directa.

Se seleccionaron sólo los cuerpos de prueba que en su superficie se encontraran indemnes ( $\sin$ irregularidades en su conformación) y aquellos cuerpos de prueba que tuvieran una diferencia no mayor a $+/-0,1 \mathrm{~mm}$ respecto del espesor $\mathrm{y}$ diámetro definido.

Para la obtención de los datos se utilizó un espectrofotómetro marca Vita Easyshade V (Modelo D-79713, Bad Säckingen, Alemania) encargado de la toma de color, un portamuestra y una caja especialmente diseñados por los investigadores para mantener las condiciones de estandarización foto lumínica ambiente $\mathrm{y}$ posicionamiento del espectrofotómetro.

El diseño de la medición mantuvo las condiciones ideales establecidas por el fabricante para la correcta toma de color del espectrofotómetro marca Vita Easyshade V. Para este estudio se utilizó el modo de "determinación del color medio" del espectrofotómetro, ya que este aparato entrega un promedio de las mediciones realizadas en una muestra.

Durante el análisis a los cuerpos de prueba se obtuvo el promedio de los 5 disparos, correspondiente a lo que sería una medición, obteniendo así una medición para cada cuerpo de prueba y su respectivo tono de las diferentes marcas comerciales estudiadas.

Se realizó una medición de color en todos los cuerpos de prueba con el espectrofotómetro Easy Shade ${ }^{\circledR}$ V, sobre un fondo negro. Para el cálculo de la diferencia de color de CIE L*a*b (E*) entre los valores obtenidos de cada cuerpo de muestra y la Escala Vita Classical, se utilizó los valores CIELab ${ }^{\circledR T M}$ de dicha escala correspondientes al año 2016.

$$
\Delta \mathrm{E}^{*}=\left\{\left(\Delta \mathrm{L}^{*}\right) 2+\left(\Delta \mathrm{a}^{*}\right) 2+\left(\Delta \mathrm{b}^{*}\right) 2\right\} 1 / 2
$$

Donde $\Delta$ son las diferencias matemáticas entre $\mathrm{L}^{*}, \mathrm{a}^{*} \mathrm{y} \mathrm{b}^{*}$ de los diferentes productos en cada 
tono. Para definir la relación entre las diferencias entre rangos colorimétricos y las diferencias de percepción visual de color, se consideraron los rangos establecidos en la Tabla I (Ttabla I).

\section{Tabla l: Valores $\Delta E$ y su interpretación.}

\begin{tabular}{|l|l|}
\hline Valores $\Delta \mathrm{E}$ & Interpretación \\
\hline$\Delta \mathrm{E}$ menor a 1 & $\begin{array}{l}\text { La diferencia de color no será } \\
\text { percibida por el ojo humano }\end{array}$ \\
\hline$\Delta \mathrm{E}$ entre 1 y 2 & $\begin{array}{l}\text { La diferencia de color será } \\
\text { percibida por un observador } \\
\text { experimentado }\end{array}$ \\
\hline$\Delta \mathrm{E}$ entre 2 y 3 & $\begin{array}{l}\text { Diferencia evidente entre color } \\
\text { de referencia y color obtenido, } \\
\text { pero gusta, es aceptable }\end{array}$ \\
\hline$\Delta \mathrm{E}$ entre 3 y 4 & $\begin{array}{l}\text { Diferencia evidente entre color } \\
\text { de referencia y color obtenido, } \\
\text { pero no perjudica al trabajo }\end{array}$ \\
\hline$\Delta \mathrm{E}$ entre 4 y 5 & $\begin{array}{l}\text { Diferencia evidente entre color } \\
\text { de referencia y color obtenido, } \\
\text { al límite de la aceptación }\end{array}$ \\
\hline Superior a 5 & $\begin{array}{l}\text { Diferencia evidente entre color } \\
\text { de referencia y color obtenido, } \\
\text { inaceptables }\end{array}$ \\
\hline
\end{tabular}

Dentro de las variables independientes se encuentran las diferentes marcas comerciales de resina compuesta que simulan dentina codificadas en tono A3 o su respectiva equivalencia, y también los valores CIE L*a*b correspondientes a cada marca.

CIE L*a*b es una clasificación del color de manera tridimensional, donde el eje ${ }^{*} \mathrm{~L}$ es de valor o brillo que va de 0 a 100 donde 0 es negro y 100 es blanco. El eje *a representa a la variación rojizo verdoso, donde los valores positivos del eje a corresponden a coloración rojiza y los valores negativos a coloración verdosa y el eje $* \mathrm{~b}$ al amarillento azulado, donde los valores positivos corresponden al color amarillo y los valores negativos al color azul. En el caso que estos ejes sean 0 es porque son acromáticos. Los valores $\mathrm{L}^{*} \mathrm{a}^{*} \mathrm{y} \mathrm{b}^{*}$ del sistema CIE $\mathrm{L}^{*} \mathrm{a} * \mathrm{~b}$ fueron obtenidos por medio de un colorímetro digital para cada muestra.

Dentro de las variables dependientes está la diferencia de color que, bajo condiciones naturales, la luz que ilumina un objeto es luz blanca, la cual es la mezcla de todos los colores del espectro visible. Un objeto presenta un color cuando su superficie es capaz de absorber cierta longitud de onda específica de la luz incidente. Cuando una o más longitudes de ondas son reflejadas, un objeto se reconoce como de un color específico. Son las diferencias matemáticas entre los valores de $\mathrm{L}^{*}, \mathrm{a}^{*} \mathrm{y} \mathrm{b}^{*}$ de los diferentes productos en cada tono. Se calcula con los promedios de los valores $\mathrm{L}^{*} \mathrm{a}^{*} \mathrm{~b} *$ de las muestras mediante la siguiente ecuación:

$\Delta \mathrm{E}^{*}=\left\{\left(\Delta \mathrm{L}^{*}\right) 2+\left(\Delta \mathrm{a}^{*}\right) 2+\left(\Delta \mathrm{b}^{*}\right) 2\right\} 1 / 2$

Para probar la hipótesis nula se realizó un análisis descriptivo de los datos y un análisis estadístico. Para el análisis estadístico se realizó una prueba de ANOVA de 2 factor (Marca y tono) con una prueba de significancia de un $0.05(\mathrm{p}=.05)$. La prueba estadístico se realizó con el programa IBM SPSS (V2; IBM Corp. Armonk, NY).

\section{RESULTADOS}

Se calcularon las medias y desviaciones estándar respectivas de las distintas marcas de resina compuesta en sus dos tonos, A2 y A3, en los dos fondos utilizados (ver tabla II). El análisis de datos se realizó separando ambos fondos para buscar la interacción que existe entre el material de resina compuesta y el color.

Se encontró una interacción de material y color estadísticamente significativa en ambos fondos, lo que quiere decir que, dependiendo del material, el color presentará un Delta E distinto.

También se elaboró una tabla resumen con todas las interacciones encontradas entre las diferentes marcas de resina compuesta (ver tabla III).

El $\Delta \mathrm{E}$ más cercano a la escala vita fue dado por Spectra A2 (1.0280), el $\Delta \mathrm{E}$ mayor fue de Filtek Z350 A3 (69.3160).

\section{DISCUSIÓN}

Los principales hallazgos del estudio se dan a partir de los $\Delta \mathrm{E}$ obtenidos en cada una de las muestras al ser medidas mediante un espectrofotómetro y contrastar dicho valor con aquellos otorgados por la escala Vita Clásica en nomenclatura CIELAB. Acorde a esto evidenciamos que existen claras diferencias entre las resinas de las marcas comerciales estudiadas y la escala Vita Clásica, ya que en el fondo negro un $71,4 \%$ de todas las muestras y en fondo 
Tabla II: Media y desviación estándar en ambos fondos según marca.

\begin{tabular}{|c|c|c|c|c|c|}
\hline & \multicolumn{2}{|c|}{ Fondo negro } & \multicolumn{2}{|c|}{ Fondo blanco } \\
\hline Marca & Color & Promedio & Desviación Std & Promedio & Desviación Std \\
\hline \multirow[t]{2}{*}{ Tetric } & A3 & 4.2880 & .89374 & 6.4160 & .54358 \\
\hline & A2 & 5.0500 & .30635 & 3.5200 & .23917 \\
\hline \multirow[t]{2}{*}{ Opallis } & A3 & 15.2320 & .69686 & 22.0240 & .71689 \\
\hline & A2 & 15.9220 & .72637 & 22.0080 & .58743 \\
\hline \multirow[t]{2}{*}{ Spectra } & A3 & 1.8800 & .31914 & 9.6720 & .42334 \\
\hline & A2 & 1.0280 & .19032 & 9.7080 & .82093 \\
\hline \multirow[t]{2}{*}{ Brilliant } & A3 & 5.2100 & .35391 & 9.8180 & .54412 \\
\hline & A2 & 6.0380 & .77147 & 11.4180 & .47499 \\
\hline \multirow[t]{2}{*}{ Empress } & A3 & 14.2800 & .47917 & 19.5400 & .76495 \\
\hline & $\mathrm{A} 2$ & 15.9740 & .75497 & 21.7180 & .24874 \\
\hline \multirow[t]{2}{*}{ Miris } & A3 & 9.1780 & .34120 & 15.5720 & .68755 \\
\hline & A2 & 8.4500 & .22661 & 13.8160 & .42747 \\
\hline \multirow[t]{2}{*}{ Z350 } & A3 & 69.3160 & .50451 & 15.2440 & .96197 \\
\hline & A2 & 3.6440 & .32176 & 14.5020 & .80335 \\
\hline
\end{tabular}

Tabla III: Análisis comparativo entre los diferentes sistemas de resina compuesta.

\begin{tabular}{|c|c|c|c|c|}
\hline & \multicolumn{2}{|l|}{ Fondo negro } & \multicolumn{2}{|c|}{ Fondo Blanco } \\
\hline Marca-tono & Marca-tono & Significancia & Marca-tono & Significancia \\
\hline \multirow[t]{3}{*}{ Tetric A3 } & Brilliant A3 & .308 & \multirow[t]{3}{*}{ - } & \multirow[t]{3}{*}{ - } \\
\hline & Tetric A2 & .611 & & \\
\hline & $\mathrm{Z} 350 \mathrm{~A} 2$ & .826 & & \\
\hline \multirow[t]{3}{*}{ Opallis A3 } & Empress A3 & .262 & Opallis A2 & 1.00 \\
\hline & Opallis A2 & .750 & \multirow[t]{2}{*}{ Empress A2 } & \multirow[t]{2}{*}{1.00} \\
\hline & Empress A2 & .651 & & \\
\hline \multirow[t]{2}{*}{ Spectra A3 } & \multirow[t]{2}{*}{ Spectra A2 } & \multirow[t]{2}{*}{.431} & Brilliant A3 & 1.00 \\
\hline & & & Spectra A2 & 1.00 \\
\hline \multirow[t]{3}{*}{ Brilliant A3 } & Tetric A3 & .380 & Spectra A3 & 1.00 \\
\hline & Tetric A2 & 1.00 & Spectra A2 & 1.00 \\
\hline & Brilliant A2 & .478 & Brilliant A2 & 0.11 \\
\hline Empress A3 & Opallis A3 & .262 & - & - \\
\hline \multirow[t]{2}{*}{ Miris S3 } & Miris S2 & .678 & Z350 A3 & 1.00 \\
\hline & & & $\mathrm{Z} 350 \mathrm{~A} 2$ & .301 \\
\hline \multirow[t]{3}{*}{$\mathrm{Z350} \mathrm{A3}$} & \multirow[t]{3}{*}{-} & \multirow[t]{3}{*}{-} & Miris S3 & 1.00 \\
\hline & & & Miris S2 & .037 \\
\hline & & & $\mathrm{Z350} \mathrm{A2}$ & .829 \\
\hline \multirow[t]{3}{*}{ Tetric A2 } & Tetric A3 & .611 & \multirow[t]{3}{*}{-} & \multirow[t]{3}{*}{-} \\
\hline & Brilliant A3 & 1.00 & & \\
\hline & Brilliant A2 & .213 & & \\
\hline \multirow[t]{2}{*}{ Opallis A2 } & Opallis A3 & .750 & Opallis A3 & 1.00 \\
\hline & Empress A2 & 1.00 & Empress A2 & 1.00 \\
\hline \multirow[t]{2}{*}{ Spectra A2 } & \multirow[t]{2}{*}{ Spectra A3 } & \multirow[t]{2}{*}{.431} & Spectra A3 & 1.00 \\
\hline & & & Brilliant A3 & 1.00 \\
\hline \multirow[t]{2}{*}{ Brilliant A2 } & Brilliant A3 & .478 & \multirow[t]{2}{*}{ Brilliant A3 } & \multirow[t]{2}{*}{0.11} \\
\hline & Tetric A2 & .213 & & \\
\hline \multirow[t]{2}{*}{ Empress A2 } & Opallis A3 & .651 & Opallis A3 & 1.00 \\
\hline & Opallis A2 & 1.00 & Opallis A2 & 1.00 \\
\hline \multirow[t]{2}{*}{ Miris S2 } & \multirow[t]{2}{*}{ Miris S3 } & .678 & Z350 A3 & .037 \\
\hline & & & $\mathrm{Z} 350 \mathrm{~A} 2$ & .893 \\
\hline $\mathrm{Z} 350 \mathrm{~A} 2$ & Tetric A3 & .826 & Miris S3 & .031 \\
\hline & & & Z30 A3 & .829 \\
\hline & & & Miris S2 & .893 \\
\hline
\end{tabular}


blanco un $92,8 \%$ están sobre un $\Delta \mathrm{E} 5$, lo cual significa que las diferencias entre sus colores reflejadas en el tinte son consideradas inaceptables clínicamente, o sea, que presentan un comportamiento muy diferente al patrón que debiesen seguir.

Por otro lado, se encuentran los que sí cumplieron con los parámetros. De estos, tenemos que en solo un 14,2\%, correspondiente a la marca Spectra en sus tonos A2 y A3, presentaron una diferencia de color que solo será percibida por un observador experimentado, esto siendo evaluado en el fondo negro. ${ }^{5}$ En un 7,1 $\%$, correspondiente a la resina Filtek Z350 en tono A2, de la muestra evaluada en fondo negro, encontramos que existe diferencia entre el tono de referencia y el obtenido que no perjudica el trabajo, lo cual se traduce en que un operador no entrenado no percibirá la diferencia de color, lo cual clínicamente no sería un gran impedimento, ya que, el paciente pudiera no notar la diferencia.

Respecto al fondo blanco obtenemos que solo el $7,1 \%$, correspondiente a la resina Tetric en tono A2, está bajo un $\Delta \mathrm{E} 5$, específicamente encontrándose en el rango de $\Delta \mathrm{E} 3-4$, lo que se traduce en que existe diferencia entre el tono de referencia y el obtenido, pero que no va a perjudicar el trabajo clínicamente.

Se consideraron un total de 35 cuerpos de prueba, los cuales fueron realizados por un solo operador, el cual fue previamente calibrado. Para evitar confusiones, se eliminaron todos aquellos cuerpos que no cumplían con los criterios de selección considerando sólo los cuerpos de prueba que en su superficie se encontraran indemnes ( $\sin$ irregularidades en su conformación) y aquellos cuerpos de prueba que tuvieran una diferencia no mayor a $+/-0,1 \mathrm{~mm}$ respecto del espesor y diámetro definido.

Además, se utilizó una caja de estandarización, cuyo diseño fue confeccionado con el fin de poder reproducir las mismas condiciones de luz ambiente y posición del espectrofotómetro para cada medición entre cuerpos de prueba.

Las marcas de resina compuesta utilizadas en este estudio fueron seleccionadas por conveniencia, por lo cual los resultados obtenidos no podrían ser generalizados a otras marcas de resina compuesta existentes en el mercado que no fueron incluidas.

En el estudio de Browning y cols., el año 2009, cuando se compararon varias muestras de color de las guías de colores Vitapan Classical con las muestras correspondientes hechas de compuestos restauradores directos, ninguna combinación de material / color resultó en un desajuste aceptable en relación con el estándar de aceptabilidad utilizado. Por lo tanto, los compuestos de resina evaluados exhibieron una pobre coincidencia en comparación con las muestras Vitapan Classical. ${ }^{6}$

Los resultados obtenidos muestran que el tono A3 de la marca Z350 3M ESPE es el que presenta mayor diferencia de color respecto a la escala Vita, en contraste con el estudio de Valdivieso y colaboradores, en donde con muestras similares obtuvieron que en un fondo negro y uno blanco, dicha resina compuesta de la misma marca y tono (A3), fue la única que no presentó variación de color con su respectivo tono del muestrario Vitapan Classical. ${ }^{7}$

Yu y Lee, explicaron que los parámetros L* a* y $b^{*}$ disminuyeron cuando se tomaron los registros sobre fondo negro, esto indica que el fondo negro no sólo influye en la luminosidad de la restauración, sino que también en el croma, por lo tanto, es esperable obtener $\Delta \mathrm{E}$ mayores a los aceptables clínicamente. ${ }^{8}$

Considerando los resultados del estudio, una buena técnica a seguir en la práctica clínica sería confeccionar una esfera polimerizada de resina compuesta posterior a haber elegido el color del diente del paciente a restaurar, y así comparar si el color se condice con la restauración que queremos lograr, ya que las resinas cambian de color al ser foto-curadas. ${ }^{9}$

Además, hay que considerar que para la práctica clínica muchas de las diferencias aquí percibidas mediante el espectrofotómetro y comparándolas con la escala Vita Clásica no llegan a ser perceptibles clínicamente o no ante un ojo no experimentado, lo cual significa que independiente de estos resultados, aun así, pueden ser satisfactorios al momento de realizar las restauraciones en los pacientes.

En relación a las limitaciones del estudio, 
encontramos que una de ellas fue razones de índole económicas, ya que no pudieron adquirir más de un lote de cada resina, sino que tuvieron que atenerse a aquellas donadas por las marcas.

Otra limitación fue la pérdida de tiempo y material al realizar los cuerpos de prueba, ya que solo había un operador calibrado el cual tenía que efectuar los cuerpos y, si estos tenían alguna falla, había que descartarlos y generar otros nuevos para poder cumplir con los parámetros establecidos para estar dentro de la muestra.

Sugerimos para otros estudios similares el utilizar más de un lote de cada jeringa de resina compuesta de las distintas marcas, ya que en este estudio encontramos que solo el de la marca Filtek Z350 3MESPE fue el que registró mayor $\Delta \mathrm{E}$. Si la muestra se abriera para estudiar más lotes de las mismas marcas, quizás se encontrarían otros resultados, en donde el $\Delta \mathrm{E}$ de esta marca no sea tan elevado ni tan diferente a las otras marcas, ya que se pudo deber a que justo ese lote no cumplía con el parámetro del tinte, pero puede que otros si lo hubieran hecho.

\section{CONCLUSIÓN}

Luego del análisis se puede concluir que ninguna de las marcas de resinas compuestas cumple con las características colorimétricas al compararlas con la escala Vita Clásica.

$\mathrm{Al}$ evaluarlas en el fondo negro el $71,4 \%$ de las resinas de tono A2 y A3 no cumplen las características colorimétricas de ser aceptable clínicamente, solo la marca Spectra Dentsply/Sirona en su tono A2 y A3, según correlación entre evaluación clínica y diferencia de color5 presentarían un comportamiento aceptable colorimétricamente frente a un observador entrenado y en segundo lugar queda la resina Filtek Z350 en su tono A2 que frente a un observador no entrenado tiene un comportamiento aceptable.

Respecto al fondo blanco el $100 \%$ de las resinas evaluadas en el tono A3 no cumplen con las características colorimétricas determinadas por Vita Clásica, y en el tono A2 el porcentaje es de un $85,7 \%$ que no cumple, siendo la excepción la resina de la línea Tetric Ivoclar Vivadent en el tono A2 que presenta un comportamiento aceptable frente a un observador no entrenado.

\section{REFERENCIAS}

[1] Steenbecker. O: Principios y bases de los biomateriales en operatoria dental estética adhesiva. Editorial Universidad de Valparaíso. Capitulo VII: Propiedades ópticas-color. Valparaíso-Chile, 2006 pp. 201-247.

[2] Tung FF, Goldstein GR, Jang S, Hittelman E.: The repeatability of an intraoral dental colorimeter. J Prosthet Dent 2002. 88: 585-90. doi: 10.1067/mpr.2002.129803.

[3] Villarroel M, Fahl N, De Sousa AM, De Oliveira OB Jr. "Direct esthetic restorations based on translucency and opacity of composite resins". J Esthet Restor Dent 2011; 23(2):73-87. doi: 10.1111/j.17088240.2010.00392.x.

[4] Yuan, J; Brewer, J; Monaco, E; Davis, E: Defining a natural tooth color space base on a 3-dimensional shade system. The Journal Prothetic Dentristry 2007; 98: 110-119. doi: 10.1016/S0022-3913(07)60044-4.

[5] Zafra Vallejo M.: Estudio experimental, in vitro, sobre la estabilidad cromática de los composites Amaris ${ }^{\circledR}$ (voco) Universidad Complutense de Madrid. 2012. pp 32-37.

[6] Browning W., Contreras R., Brackett M., Brackett W. Color differences: Polymerized composite and corresponding Vitapan Classical shade tab. Journal of dentistry 2009; 37: 34-39. doi: 10.1016/j.jdent.2009.05.008.

[7] Ryan EA., Tam LE., McComb D.: Comparative Translucency of esthetic composite resin restorative materials. J Can Dent Assoc 2010; 76:a84. PMID: 20719098

[8] Kim Jong-Gil; Yu B; Lee YK. Correlations between Color Differences Based on Three Color-Difference Formulas Using Dental Shade Guide Tabs. Journal of prosthodontics: official journal of the American College of Prosthodontists 2008; 18 . 135-40. doi: 10.1111/j.1532-849X.2008.00393.x

[9] Valdivieso J, et al; Influencia del grosor de una resina compuesta para técnica estratificada, en la expresión de su color final. Revista dental de Chile 2016; 107. 52-55

\section{CÓMO CITAR ESTE ARTÍCULO}

Sarmiento J, Morales J, Hidalgo L, Leiva I. Evaluación instrumental colorimétrica de resinas compuestas que imitan dentina en comparación a escala vita clásica. Appli. Sci. Dent. 2020; 1(1): 47-53. DOI: 10.22370/asd.2020.1.1.2113 